\title{
A sustentabilidade hídrica na bacia do rio Guamá, Amazônia Oriental/Brasil
}

\section{Water sustainability of the Guamá river basin, Eastern Amazonia/Brazil}

\author{
Nivia Cristina Vieira Rocha \\ Aline Maria Meiguins de Lima ${ }^{2}$
}

\section{Palavras-chave \\ Indicadores \\ Bacia hidrográfica \\ Manejo de bacias}

\section{Keywords}

Indicators

Watershed

Management

\begin{abstract}
Resumo
A bacia hidrográfica do rio Guamá abrange 19 municípios localizados no nordeste do Pará, com grande diversidade de formas de uso da terra e a maior densidade demográfica do estado. Nesta pesquisa foi avaliada sua sustentabilidade hídrica, a partir dos aspectos hidrológicos, ambientais, sociais e de gestão, nas suas 8 sub-bacias componentes. O indicador hidrológico demonstrou um comportamento mediano para às sub-bacias; o ambiental destacou a fragilidade da cobertura vegetal resiliente; o social apresentou o pior desempenho; e o de gestão indicou a necessidade do fortalecimento institucional. A bacia do rio Guamá no geral obteve um índice de sustentabilidade intermediário, porém os resultados parciais apontam para necessidade de medidas voltadas ao planejamento estratégico e ao manejo, para minimizar as pressões sobre a vegetação remanescente, reforçar a capacidade institucional e melhorar a qualidade dos recursos e de vida da população, com a intenção de potencializar a sustentabilidade da bacia.
\end{abstract}

\begin{abstract}
The Guamá River basin covers 19 municipalities located in northeastern Pará, with great diversity of land use forms and the highest population density of the state. In this research, its water sustainability was evaluated, based on the hydrological, environmental, social and management aspects, in its 8 sub-basins. The hydrological indicator showed a medium behavior for the subbasins; the environmental highlighted the fragility of the resilient vegetal cover; the social performance presented the worst performance; and the management pointed out the need for institutional strengthening. The Guamá river basin in general obtained an intermediate sustainability index, but the partial results indicated that for measures geared to the strategic planning and management, to minimize pressures on the remaining vegetation, enhancing institutional capacity and improving the quality of life of the population and resources, with the intention of enhancing the sustainability of the basin.
\end{abstract}

${ }^{1}$ Universidade Federal do Pará, Belém, Pará, Brasil. niviavieira.ciamb@gmail.com

2 Universidade Federal do Pará, Belém, Pará, Brasil. ameiguins@gmail.com 


\section{INTRODUÇÃO}

O significativo crescimento demográfico gerou nos últimos anos uma maior demanda sobre os recursos hídricos, tanto para suprir as necessidades da população como também da indústria e, além disso, as alterações de áreas com cobertura vegetal devem provocar, de médio a longo prazo, modificações no potencial hídrico (PRATES; BACHA, 2011). Com isso é ressaltada a necessidade de avanços institucionais e tecnológicos para a recuperação e proteção dos sistemas hídricos, além de novas visões para a gestão preventiva, integrada e adaptativa (MARQUES, 2017).

O consumo per capita do recurso aumenta de acordo com a melhora de renda da sociedade, sendo que a água é empregada em diversos usos os quais estão diretamente relacionados à economia (regional, nacional e internacional); os mais comuns e frequentes são para uso doméstico, irrigação, uso industrial e hidroeletricidade, o que reforça a necessidade da realização de um manejo apropriado para os recursos hídricos (RIBEIRO; PIZZO, 2011).

A partir da década de 1980, com a modernização dos modelos de gestão da água, passou-se a incorporar o conceito de sustentabilidade, fazendo com que a gestão ambiental e hídrica tivesse sua importância reforçada por meio das políticas públicas de desenvolvimento (CARVALHO, 2014). Manter a sustentabilidade hídrica é de fundamental importância já que a mesma leva em consideração a disponibilidade quantitativa e qualitativa de acordo com um acesso equilibrado, dentro dos usos e das exigências de cada bacia hidrográfica (TRINDADE; SCHEIBE, 2019). A sustentabilidade hídrica implica em conservar um equilíbrio dinâmico entre a oferta e a demanda por água, de modo que os mananciais sejam utilizados a taxas iguais ou inferiores a sua resiliência (SOOD; RITTER, 2011).

Dentre as formas de subsidiar uma gestão dos recursos hídricos, capaz de contribuir para a busca do desenvolvimento sustentável, têm-se as ferramentas adequadas de medir o desempenho dos sistemas hídricos e ambientais (CARVALHO et al., 2011). Portanto, o desenvolvimento de um índice de sustentabilidade hídrica corresponde a uma análise multidisciplinar tratando de vários aspectos de integração de diversos parâmetros (VIEIRA; STUDART, 2009).

Embora existam vários aspectos ambientais de sustentabilidade e índices de escassez de água, estes não são projetados para avaliar a bacia hidrográfica contemplando a integração entre seus diversos componentes; neste contexto, a Organização Internacional Programa de Hidrologia (IHP) da Organização das Nações Unidas para a Educação, a Ciência e a Cultura (UNESCO) iniciou e apoiou a pesquisa e desenvolvimento de índices de acordo com a estratégia de desenvolvimento de cada local de aplicação (CORTÉS et al., 2012).

A construção de indicadores de sustentabilidade hídrica tem sua base nos princípios de controle da oferta pelo 
gerenciamento e de monitoramento da disponibilidade, segundo a FAO (2017), esta premissa é associada a percepção de consumo sustentável e de controle da escassez e poluição das águas.

Os métodos de avaliação destes indicadores, variam de acordo com os objetivos propostos envolvendo conceitos como sustentabilidade, vulnerabilidade, pegada ecológica e parâmetros socioambientais (SULLIVAN et al., 2003; BÖHRINGER; JOCHEM, 2007; BLANC et al., 2008; EMERSON et al., 2010). De maneira geral são envolvidos conceitos estatísticos como média aritmética mediante padronização, média ponderada por pesos iguais, distribuição de pesos baseada em análises estatísticas e a partir de consultas a especialistas (WELSCH et al., 2005; NARDO et al., 2008; RICKWOOD; CARR, 2009). A seleção do melhor método depende das características do modelo e dos parâmetros adotados (JUWANA et al., 2012).

Dentre os índices de comportamento hidrológico (mais voltados a avaliação da disponibilidade hídrica a partir de fatores hidrometeorológicos) pode-se destacar (KEYANTASH; DRACUP, 2004; BLAIN; BRUNINI, 2007; SOUSA et al., 2010; FARO et al., 2019): Índice de Precipitação Padronizado; Índices de Seca de Palmer; Índice de Anomalia de Precipitação; e Índice Agregado de Seca.

Além destes, podem ser agrupados aqueles que envolvem parâmetros biofísicos e socioeconômicos (SULLIVAN, 2002; XU et al., 2002; BOYACIOGLU, 2007; CHAVES; ALIPAZ, 2007; TEJADA-GUIBERT et al.,
2015; FERREIRA et al., 2016; SILVA et al., 2017), tais como: Índice Integrado de Gestão de Recursos Hídricos; Índice de Sustentabilidade Hídrica; Índice de Potencial Degradação dos Recursos Hídricos e Índice de Qualidade das Águas.

Os índices compostos (WILLET et al., 2019) são construídos pela agregação de indicadores, sendo seus resultados diretamente aplicáveis aos tomadores de decisão, uma vez que grandes quantidades de informações podem ser condensadas em valores mais gerenciáveis e comparáveis, para sua melhor aplicação e representatividade.

De acordo com HTCF (2003), indicadores de sustentabilidade de bacias hidrográficas devem atender a alguns critérios básicos para que possam ser úteis, sendo assim, devem ser: disponíveis e de fácil acesso, compreensíveis, confiáveis, relevantes e integradores. A aplicação de um índice de sustentabilidade hídrica, abrangendo diferentes aspectos e respostas socioeconômicas e ambientais, tornase útil para a verificação do nível de sustentabilidade das bacias hidrográficas, permitindo a obtenção de um conjunto de indicadores, além possibilitar a elaboração de instrumentos capazes de identificar empecilhos que dificultam a gestão (CHAVES; ALIPAZ, 2007).

A partir deste contexto, a bacia hidrográfica do rio Guamá localizada na Região Hidrográfica Costa Atlântica Nordeste do estado do Pará, abrange 19 municípios no domínio do Bioma Amazônico inseridos na área de influência do chamado "Arco do 
Desmatamento da Amazônia, tendo apresentado nos últimos anos um avanço no processo de desflorestamento" (RIVERO et al., 2009; SILVA et al., 2013; BARROSO et al., 2015).

A relevância da análise da bacia do rio Guamá no contexto hídrico do nordeste paraense indica a necessidade de uma percepção a respeito da composição e estrutura de sua paisagem (BEZERRA et al., 2011; SANTOS et al., 2016), além da importância da aquisição de informações pertinentes para a análise da qualidade de vida da população desta área, as quais possibilitem o emprego de ações voltadas ao manejo e gestão da mesma.

O objetivo desta pesquisa foi aplicar um índice de sustentabilidade hídrica, adaptado de Chaves e Alipaz (2007), para a bacia do Rio Guamá. Onde a análise espacial de paisagem foi associada aos parâmetros de escoamento superficial para compor o eixo hidroambiental e os aspectos ligados à qualidade de vida da população e voltados para a capacidade institucional dos municípios componentes, formam a componente social e de gerenciamento.

Os resultados obtidos visam a obtenção de um conjunto de indicadores específicos para a bacia do rio Guamá (e municípios componentes), além possibilitar a elaboração de ferramentas que identifiquem os entraves para a gestão hídrica na região e que possam contribuir para o planejamento do uso e ocupação da terra, na perspectiva de hidroterritório amazônico, conforme Becker (2009) e Aragón (2018).

\section{MATERIAL E MÉTODOS}

\section{Área de Estudo}

A bacia hidrográfica do rio Guamá está situada no nordeste do estado do Pará, abrangendo uma área com cerca de $11870 \mathrm{~km}^{2}$, representando 1\% da área do estado (Figura $1)$.

A mesma está inserida na Região Hidrográfica Costa Atlântica - Nordeste, segundo a resolução n ${ }^{\circ}$ 04/2008 do Conselho de Recursos Hídricos do Estado do Pará, mostrando-se como área de grande circulação econômica composta por 19 municípios que se caracterizam devido o desenvolvimento de atividades voltadas para indústria, mineração, comércio e agropecuária. São estes: Capitão Poço, Garrafão do Norte, Irituia, São Miguel do Guamá, Bujarú, Santa Luzia do Pará, Concórdia do Pará, Santa Izabel do Pará, Inhangapi, Castanhal, São Domingos do Capim, Ourém, Mãe do Rio, Belém, Acará, Bonito, Benevides, Marituba e Ananindeua, que totalizam cerca de 2.700 .000 habitantes segundo o último censo (BRASIL, 2010). 
Figura 1 - Localização da área de estudo.

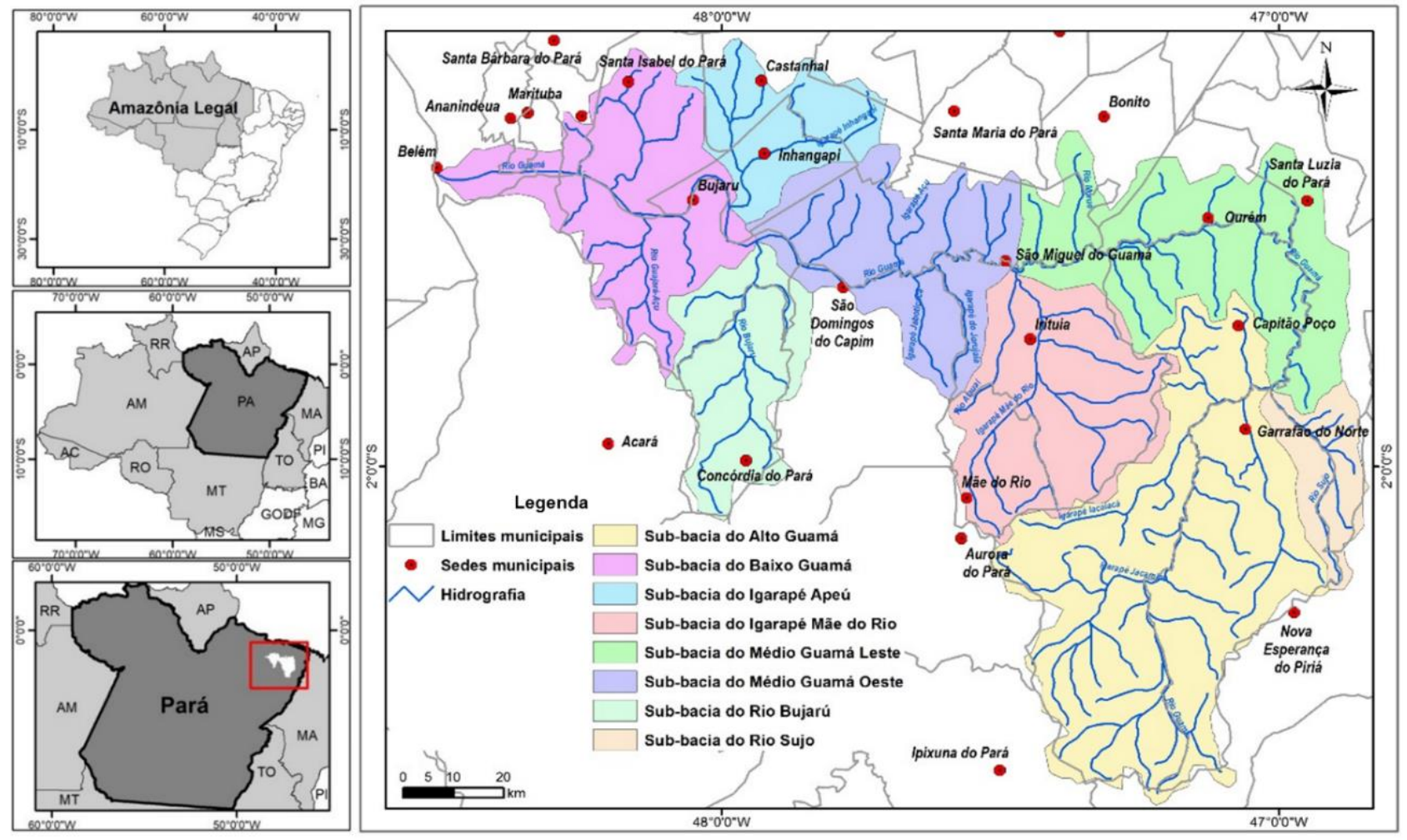

Fonte: Lab. de Estudos e Modelagem Hidroambientais (LEMHA), 2018. Org.: Autor, 2018.

\section{Avaliação da sustentabilidade hídrica}

O cálculo do índice de sustentabilidade hídrica

- ISH para a bacia hidrográfica do rio Guamá foi uma adaptação (para a condição de Estado) da metodologia aplicada por Chaves e Alipaz (2007), a qual leva em consideração aspectos hidrológicos $(\mathrm{H})$, ambientais $(\mathrm{A})$, sociais (S) e de gestão (G), já que a sustentabilidade de uma bacia é um processo dinâmico.

Para a aplicação do índice em bacias como a do Guamá, com uma extensão superior a $10.000 \mathrm{~km}^{2}$, é mais viável que a mesma seja dividida em sub-bacias e tenha o valor do mesmo calculado de forma ponderada.

Com isto, a bacia do rio Guamá foi dividida em 8 sub-bacias para análise: Baixo Guamá, Igarapé Apeú, Rio Bujarú, Médio
Guamá setor oeste, Médio Guamá setor leste, Igarapé Mãe do Rio, Rio Sujo e Alto Guamá, delimitadas de acordo com o sistema fluvial considerando o rio principal e seus afluentes.

Para analisar a variável ambiental (A) foi elaborado uma carta de uso e cobertura da terra, com a definição de 8 classes adaptadas segundo o Manual de Uso da Terra do IBGE (BRASIL, 2013): área vegetada, agricultura, áreas de ocupação, solo descoberto, pastagem, massa d'água, áreas não observadas e outros.

Este foi gerado por meio da técnica de classificação supervisionada e multiespectral, baseada no método de Máxima Verossimilhança (MaxVer), realizada pixel a pixel, por meio do software ENVI em imagens ortorretificadas de satélites do sistema RapidEye (de alta resolução, em um total de 
43 cenas, datadas de 29/06/2011, 28/07/2011, 04/08/2011, 23/10/2011, 31/07/2012, 02/08/2012, 13/09/2012, 24/10/2012, 01/08/2013, 04/09/2013, 17/08/2014 e 25/11/2014, as quais contém 5 bandas espectrais, estas foram obtidas por meio do Geo Catálogo do Ministério do Meio Ambiente (MMA); as cenas selecionadas apresentaram melhores condições relacionadas a menor índice de nebulosidade, o que favorece na análise e classificação dos alvos), o qual possibilitou a realização da análise de paisagem e quantificação das áreas que possuem cobertura florestal (relação entre área total de cada sub-bacia e a área ocupada pela cobertura florestal), com a validação do resultado por levantamentos de campo.

Em relação a variável hidrológica $(\mathrm{H})$ foi utilizado o modelo hidrológico curva número $\mathrm{CN}$, que é empregado para estimar a dinâmica do escoamento superficial nas sub-bacias, de acordo com a análise dos tipos de uso e cobertura da terra. Foram adotados os procedimentos do Soil Conservation Service (SCS), adaptados por Calzavara e Fernandez (2015) para condições brasileiras. Os valores de precipitação foram obtidos a partir dos dados de 11 estações pluviométricas localizadas na área de influência direta da bacia hidrográfica do rio Guamá (série de 1985 a 2015), disponibilizados no software Hidro 1.2 da Agência Nacional de Águas (ANA). Estes foram espacializados de acordo com o método do Polígono de Thiessen, o qual atribuiu um fator de ponderação aos totais precipitados em cada pluviômetro à área de influência de cada um (CORREIA; RIBEIRO; BAPTISTA, 2015).

$\mathrm{Na}$ análise da variável social (S) foram consideradas as informações referentes às questões sociais dos municípios que compõem as sub-bacias levando em consideração aspectos institucionais, políticos e socioeconômicos vinculados à população, os quais afetam diretamente a gestão dos recursos hídricos, como o índice de desenvolvimento humano por município (IDHM) e o índice de Gini municipal (BRASIL, 2010), além do índice de consumo de água e índice de atendimento total no abastecimento de água por município segundo o Sistema Nacional de Informações sobre o Saneamento SNIS (BRASIL, 2016). Estes dados foram verificados também junto as informações coletadas nas secretarias municipais e junto ao governo do Estado.

Após a obtenção das variáveis foi realizada a média aritmética para o cálculo do indicador social para cada município (Equação 1). Este indicador colaborou para a comparação dos municípios e análise dos mesmos. Em seguida, foi utilizada a média ponderada para que os indicadores sociais de cada município fossem espacializados nas suas respectivas sub-bacias, ou seja, levou-se em consideração a área de cada município dentro das sub-bacias (Equação 2). Com isso, obtevese um indicador social para cada sub-bacia, que foi utilizado para a determinação do ISH. Onde: $\mathrm{S}_{\mathrm{mi}}=$ indicador social por município; $\mathrm{S}_{\mathrm{i}}$ $=$ variáveis analisadas; $\mathrm{n}=$ número de variáveis; $\mathrm{S}=$ indicador social na sub-bacia; $\mathrm{A}_{\mathrm{i}}$ = área do município dentro da sub-bacia (ha); 
$\mathrm{A}=$ área total da sub-bacia (ha).

$$
\begin{aligned}
& \mathrm{S}_{m i}=\frac{\sum \mathrm{s}_{\mathrm{i}}}{\mathrm{n}} \\
& \mathrm{S}=\frac{\sum_{\mathrm{i}=1}^{\mathrm{n}} \mathrm{A}_{\mathrm{i}} \mathrm{S}_{\mathrm{mi}}}{\mathrm{A}}
\end{aligned}
$$

E por fim a análise da variável de gestão (G) admitindo à média aritmética e ponderada, assim como na variável anterior, referente as características que influenciam na capacidade de gestão ambiental dos municípios que integram as sub-bacias (Tabela 1). As mesmas correspondem a 13 atributos a respeito dos municípios, adquiridas no banco de dados do
IBGE, do SNIS, do Programa Municípios Verdes e dos sites das Prefeituras Municipais. Para cada uma destas, foi atribuído um peso de 0 a 1 , para que as condições de gestão dos municípios pudessem ser analisadas e comparadas, onde 0 significa condições ruins e 1 condições boas.

Para a realização o cálculo do ISH, a partir da seleção dos indicadores os quais se enquadram nos aspectos avaliados $(\mathrm{H}, \mathrm{A}, \mathrm{S}$, G), cada um deles foi dividido em cinco pontuações de escala $(0 ; 0,25 ; 0,50 ; 0,75$ e 1$)$ a fim de simplificar a estimativa, sendo que 0 representa as piores condições e 1 para as melhores condições (Tabela 2).

Tabela 1. Parâmetros para a avaliação da gestão dos municípios.

\begin{tabular}{|c|c|c|c|c|c|}
\hline \multicolumn{6}{|c|}{ Classificação $=$ Peso $/$ Variáveis } \\
\hline$\leq 10 \%=0$ & $10 \%<G \leq 20 \%=0,25$ & \multicolumn{2}{|c|}{$20 \%<G \leq 30 \%=0,5$} & $30 \%<G \leq 50 \%=0,75$ & $>50 \%=1$ \\
\hline \multicolumn{6}{|c|}{ - Grau de Cobertura pelo Cadastro Ambiental Rural (CAR) } \\
\hline \multicolumn{2}{|c|}{ Individual $=1$} & \multicolumn{2}{|c|}{ Associada $=0,5$} & \multicolumn{2}{|c|}{ Ausente $=0$} \\
\hline \multicolumn{6}{|c|}{ - Secretaria do Meio Ambiente } \\
\hline Alto 1 & acto $=1$ & \multicolumn{2}{|c|}{ Baixo Impacto $=0,5$} & \multicolumn{2}{|c|}{$N \tilde{a} o=0$} \\
\hline \multicolumn{6}{|c|}{ - Realiza Licenciamento Ambiental } \\
\hline \multicolumn{3}{|c|}{ Existente $=1$} & \multicolumn{3}{|c|}{ Inexistente $=0$} \\
\hline \multicolumn{3}{|c|}{ - Legislação Ambiental } & \multicolumn{3}{|c|}{ - Conselho Municipal de Meio Ambiente } \\
\hline \multicolumn{3}{|c|}{$\operatorname{Sim}=1$} & \multicolumn{2}{|r|}{$N \tilde{a} o=0$} & \\
\hline \multicolumn{3}{|c|}{$\begin{array}{l}\text { - Participação no Programa Municípios Verdes } \\
\text { - Acesso às informações sobre gestão ambiental via } \\
\text { internet } \\
\text { - Realiza ações de educação ambiental no município } \\
\text { - Possui Plano Diretor Municipal }\end{array}$} & \multicolumn{3}{|c|}{$\begin{array}{l}\text { - Possui Plano de Saneamento Básico } \\
\text { - Possui Política de Saneamento } \\
\text { - Possui habilitação para a gestão ambiental } \\
\text { - Possui Fundo Municipal de Meio } \\
\text { Ambiente }\end{array}$} \\
\hline
\end{tabular}

Classificação $=$ Peso $/$ Variáveis

Org.: do Autor, 2018. 
Tabela 2. Variáveis para a aplicação do ISH.

\begin{tabular}{|c|c|c|c|}
\hline Indicador & Parâmetros de estado definidos nesta proposta & $\begin{array}{l}\text { Nível definidos nesta } \\
\text { proposta }\end{array}$ & $P_{W}$ \\
\hline \multirow{5}{*}{ Hidrológico } & \multirow{5}{*}{$\begin{array}{l}\text { Potencial de escoamento a partir do modelo Curva } \\
\text { Número - CN }\end{array}$} & $\leq 0,1$ & 0,00 \\
\hline & & $0,1<\mathrm{H} \leq 0,25$ & 0,25 \\
\hline & & $0,25<\mathrm{H} \leq 0,50$ & 0,50 \\
\hline & & $0,50<\mathrm{H} \leq 0,75$ & 0,75 \\
\hline & & $>0,75$ & 1,00 \\
\hline \multirow{5}{*}{ Ambiental } & \multirow{6}{*}{$\begin{array}{c}\% \text { área total da bacia e a área ocupada por cobertura } \\
\text { florestal }\end{array}$} & $\leq 5 \%$ & 0,00 \\
\hline & & $5 \%<\mathrm{A} \leq 15 \%$ & 0,25 \\
\hline & & $15 \%<\mathrm{A} \leq 30 \%$ & 0,50 \\
\hline & & $30 \%<\mathrm{A} \leq 45 \%$ & 0,75 \\
\hline & & $\mathrm{A}>45 \%$ & 1,00 \\
\hline \multirow{5}{*}{ Sociais } & & $\leq 0,5$ & 0,00 \\
\hline & \multirow{4}{*}{$\begin{array}{l}\text { Baseado no IDHM, Índice de Gini e de saneamento } \\
\text { segundo o SNIS - Sistema Nacional de Informações } \\
\text { Sobre Saneamento. }\end{array}$} & $0,5<\mathrm{S} \leq 0,6$ & 0,25 \\
\hline & & $0,6<\mathrm{S} \leq 0,75$ & 0,50 \\
\hline & & $0,75<\mathrm{S} \leq 0,9$ & 0,75 \\
\hline & & $>0,9$ & 1,00 \\
\hline \multirow{5}{*}{ Gestão } & \multirow{5}{*}{$\begin{array}{l}\text { Capacidade institucional da bacia: nível de organização } \\
\text { do sistema de gestão ambiental }\end{array}$} & $\leq 10 \%$ & 0,00 \\
\hline & & $10 \%<\mathrm{G} \leq 20 \%$ & 0,25 \\
\hline & & $20 \%<\mathrm{G} \leq 30 \%$ & 0,50 \\
\hline & & $30 \%<\mathrm{G} \leq 50 \%$ & 0,75 \\
\hline & & $>50 \%$ & 1,00 \\
\hline
\end{tabular}

Org.: do Autor, 2018.

O índice de sustentabilidade hídrica corresponde à somatória das variáveis segundo cada parâmetro considerado em razão do total de variáveis adotadas (Equação 3). Todos os indicadores possuem o mesmo peso, já que nenhuma das variáveis é considerada mais importante do que outra, e sim complementares para que haja a sustentabilidade da bacia, sendo: aspectos hidrológicos $\left(p_{w H}\right)$, ambientais $\left(p_{w A}\right)$, sociais $\left(p_{w S}\right)$ e de gestão $\left(p_{w G}\right)$, valorados de 0 a $1(0$ condições pobres e 1 condições ótimas); $\mathrm{n}_{i}$, representa as variáveis consideradas (Tabela 2). Após a obtenção dos resultados, os mesmos foram classificados para cada sub-bacia de acordo com a Tabela 3. Com a análise dos resultados do índice de sustentabilidade para cada sub-bacia, foi aplicada uma média ponderada em relação à área, para a obtenção do ISH para toda a bacia hidrográfica do rio Guamá.

$$
I S H=\sum p_{w i} / \sum n_{i}
$$

A análise ponderada por igual peso facilita a estruturação em dimensões dos indicadores, admitindo que estes tenham características intrínsecas com a gestão das águas, sejam mensuráveis, de acessível verificação e passíveis de comparação; reduzindo efeitos de redundância entre as variáveis selecionadas (CARVALHO et al., 2013). Desta forma, permite a integração de critérios e opções heterogêneas simultaneamente, como por exemplo, na avaliação da sustentabilidade hídrica. Fatores 
estes destacados por Flores-Alsina et al. (2010) na avaliação do índice de qualidade das águas e por Chuang et al. (2018) na avaliação de indicadores hidroambientais em sistemas costeiros.

Tabela 3. Classificação dos valores do ISH.

\begin{tabular}{cc}
\hline ISH (0,00-1,00) & Desempenho \\
$\leq 0,20$ & Ruim / insustentável \\
0,21 a 0,40 & Pobre / potencialmente \\
0,41 a 0,60 & insustentável \\
$0,61-0,80$ & Bódio / intermediário \\
$\geq 0,81$ & Muitencialmente \\
sustentável \\
\hline
\end{tabular}

Fonte: Adaptado de Carvalho et al., (2011).

De forma complementar, visando a análise integrada, os resultados foram apresentados no formato cartográfico e utilizando a análise de agrupamento. A metodologia de clustering trata-se de uma análise estatística multivariada que possibilita a identificação de grupos com características homogêneas (k-medias), considerando cálculos matemáticos de proximidade (similaridade) a todos os pares de objetos e entre cada objeto e os subgrupos, de tal forma que as distâncias entre os membros de um subgrupo sejam mínimas e a distâncias entre os subgrupos sejam máximas (YOSHIMITANAKA et al., 2015). Os resultados são apresentados como agrupamentos e heatmaps (matrizes de cores), onde cada célula corresponde à posição ocupada pelo valor de uma determinada variável em uma unidade de análise.

\section{RESULTADOS E DISCUSSÃO}

\section{Indicador ambiental}

A partir da análise da carta de uso e ocupação da terra, pode-se identificar o porcentual referente às áreas que possuem vegetação (Tabela 4), e por meio deste resultado identificar quais as sub-bacias que apresentam melhor e pior representatividade em relação ao indicador ambiental.

Observa-se que as sub-bacias que apresentam melhor estágio de conservação, com a ocorrência de áreas vegetadas são o Baixo Guamá (57,87\%) e o Rio Bujarú (55,51\%). As que possuem maior presença de áreas alterdas são representadas pelo Igarapé Mãe do Rio (24,07\%) e Alto Guamá (29,08\%).

Tabela 4. Área correspondente a cobertura vegetal nas sub-bacias analisadas.

\begin{tabular}{lccc}
\hline \multicolumn{1}{c}{ Sub-bacia } & Área da sub-bacia (ha) & $\begin{array}{c}\text { Área total de } \\
\text { vegetação (ha) }\end{array}$ & $\begin{array}{c}\text { Área total de } \\
\text { vegetação (\%) }\end{array}$ \\
\hline Sub-bacia do Alto Guamá & $331.639,30$ & $96.444,02$ & 29,08 \\
Sub-bacia do Igarapé Apeú & $74.737,99$ & $29.318,04$ & 39,23 \\
Sub-bacia do Médio Guamá Oeste & $142.137,30$ & $53.676,70$ & 37,76 \\
Sub-bacia do Baixo Guamá & $163.960,76$ & $94.885,30$ & 57,87 \\
Sub-bacia do Igarapé Mãe do Rio & $155.244,52$ & $37.359,57$ & 24,07 \\
Sub-bacia do Médio Guamá Leste & $191.134,49$ & $66.943,62$ & 35,02 \\
Sub-bacia do Rio Bujarú & $99.019,23$ & $54.964,38$ & 55,51 \\
Sub-bacia do Rio Sujo & $46.012,53$ & $17.046,30$ & 37,05 \\
\hline \multicolumn{1}{c}{ Total } & $1.203 .886,12$ & $450.637,93$ & 37,43 \\
\hline
\end{tabular}

Org.: do Autor, 2018. 
Merece destaque, que a maior concentração de áreas alteradas está associada a municípios que apresentam características voltadas ao agronegócio, com pequenos e médios produtores que suprem as necessidades do estado e grandes produtores que atendem o restante do Brasil além do mercado externo (REBELLO et al., 2011).

Os resultados obtidos estão de acordo com o que Watrin et al. (2009) destacam para a região do nordeste paraense, com as pastagens sendo o padrão dominante do uso da terra. O mesmo comportamento foi observado por Pereira et al. (2015) para a bacia do Igarapé Apeú; e por Nascimento e Fernandes (2017) para uma sub-bacia do Alto Guamá.

A cobertura vegetal é de fundamental importância para o funcionamento de diversos processos que ocorrem em um ecossistema, como por exemplo, os processos de infiltração ou escoamento, balanço de energia, manutenção de condições do clima, dentre outros. Mudanças na cobertura vegetal causadas pelo desmatamento ou implantação de atividades agropecuárias podem acarretar diversos impactos ambientais negativos, como assoreamento de rios, compactação do solo, desencadeamento de processos erosivos, dentre outros (SILVA et al., 2017).

\section{Indicador hidrológico}

Com a aplicação do método Curva Número, foram obtidos os valores de escoamento para cada uma das sub-bacias analisadas (Tabela 5). $\mathrm{O}$ escoamento superficial das sub-bacias varia entre 28 a $33 \%$ dos valores de precipitação incidente, sendo que a sub-bacia que obteve maior resultado foi a do rio Bujarú e a que teve menor representatividade foi a do Alto Guamá.

Tabela 5. Escoamento superficial médio por sub-bacia.

\begin{tabular}{lccc}
\hline \multicolumn{1}{c}{ Sub-bacia } & $\begin{array}{c}\text { Precipitação } \\
\text { (acumulado anual } \\
\text { do período) }(\mathbf{m m})\end{array}$ & $\begin{array}{c}\text { Escoamento } \\
\mathbf{( m m )}\end{array}$ & $\begin{array}{c}\text { Escoamento em } \\
\text { relação à precipitação } \\
\text { (\%) }\end{array}$ \\
\hline Sub-bacia do Rio Bujarú & $2.462,78$ & 814,12 & 33,06 \\
Sub-bacia do Baixo Guamá & $2.642,59$ & 767,70 & 29,05 \\
Sub-bacia do Igarapé Apeú & $2.425,84$ & 733,17 & 30,22 \\
Sub-bacia do Médio Guamá Oeste & $2.331,86$ & 680,46 & 29,18 \\
Sub-bacia do Igarapé Mãe do Rio & $2.392,43$ & 683,74 & 28,58 \\
Sub-bacia do Médio Guamá Leste & $2.224,02$ & 668,42 & 30,05 \\
Sub-bacia do Rio Sujo & $2.179,63$ & 610,62 & 28,01 \\
Sub-bacia do Alto Guamá & $2.066,94$ & 578,39 & 27,98 \\
\hline
\end{tabular}

Org.: do Autor, 2018.

A bacia do rio Guamá é composta basicamente por cinco tipos de solos dominantes, o Latossolo Amarelo, Latossolo Concrecionário, Neossolo Flúvico, Neossolos
Quartzarênicos e Argissolo Vermelho Amarelo, onde os Latossolos Amarelos ocupam maior parte desta, representando 84,91\%.

O Latossolo Amarelo se caracterizam 
como profundos, porosos e com textura elevada, além de uma moderada taxa de infiltração, resistência e tolerância à erosão, contribuindo para os valores medianos de escoamento em todas as sub-bacias. Essas condições favorecem a fertilidade do solo, pois quanto menor for o escoamento, menor será o arraste da fração granulométrica mais fina do solo (FEITOSA et al., 2010).

O comportamento evidenciado na Tabela 5 indica que o melhor enquadramento da bacia, segundo os parâmetros físico-hídricos, de tipo de solo dominante está entre os Grupos A e B, que classificam-se como (SARTORI et al., 2015): Grupo A - produzem baixo escoamento e alta infiltração, solos muito profundos $(>2,0 \mathrm{~m})$, com alta permeabilidade e baixa erodibilidade; Grupo B - menos permeáveis e maior potencial em gerar escoamento, consistindo principalmente de solos moderadamente profundos a profundos. Tais características possibilitam entender melhor a dinâmica do escoamento superficial na bacia. E consequentemente a avaliação do potencial de rendimento de água, contribuindo também para o planejamento de medidas capazes de conservar as características do solo e água, redução de processos de assoreamento e riscos de inundações (MUÑOZ-ROBLES et al., 2011).

\section{Indicador social}

A análise se deu sobre os parâmetros relacionados aos municípios que compõem a bacia do rio Guamá, como o índice de desenvolvimento humano municipal, o índice de Gini e dois índices do Sistema Nacional de Informações sobre o Saneamento, relacionados ao abastecimento e consumo de água. A partir destes valores médios por município, foram determinados resultados para os indicadores sociais das sub-bacias, sendo realizada por meio da média ponderada, onde foram levadas em consideração as áreas dos municípios em relação as suas respectivas sub-bacias (Tabela $6)$.

Observa-se que a sub-bacia que possui maior destaque em ralação a este indicador é a do Igarapé Mãe do Rio, com valor de 0,66. Esta é composta pelos municípios de Capitão Poço, São Domingos do Capim, Irituia e Mãe do Rio, que se destacam principalmente com relação ao abastecimento de água.

Tabela 6. Indicador Social por sub-bacia.

\begin{tabular}{lc}
\hline \multicolumn{1}{c}{ Sub-bacia } & $\begin{array}{c}\text { Indicador } \\
\text { Social }\end{array}$ \\
\hline Sub-bacia do Igarapé Mãe do Rio & 0,66 \\
Sub-bacia do Médio Guamá Oeste & 0,57 \\
Sub-bacia do Alto Guamá & 0,57 \\
Sub-bacia do Rio Sujo & 0,56 \\
Sub-bacia do Baixo Guamá & 0,55 \\
Sub-bacia do Rio Bujarú & 0,51 \\
Sub-bacia do Igarapé Apeú & 0,49 \\
Sub-bacia do Médio Guamá Leste & 0,48 \\
\hline *Não existem informações referentes ao índice. \\
Fonte: Brasil (2010); Brasil (2016). Org.: do \\
Autor, 2018.
\end{tabular}

E a sub-bacia que menos se destaca é a do médio Guamá setor leste, com valor de 0,48, composta pelos municípios de Bonito, Capitão Poço, Irituia, Ourém, Santa Luzia do Pará e São Miguel do Guamá, sendo que dois destes não possuíam informações referentes a dois dos índices analisados (Bonito e São Miguel do 
Guamá).

\section{Indicador de gestão}

A análise da gestão dos municípios foi realizada a partir da média dos valores obtidos por meio da atribuição do peso para as 13 variáveis analisadas (Tabela 1), referente a presença ou grau de cobertura da(o): legislação ambiental, conselho municipal de meio ambiente, licenciamento ambiental, Secretaria do Meio Ambiente, Cadastro Ambiental Rural (CAR), Programa Municípios Verdes, gestão ambiental via internet, educação ambiental no município, plano diretor municipal, plano de saneamento básico, política de saneamento, gestão ambiental e Fundo Municipal de Meio Ambiente. A partir destas foi gerado um valor referente ao indicador de gestão para cada município e em seguida ponderado para a área das sub-bacias (Tabela 7).

Tabela 7. Indicador de Gestão por sub-bacia.

\begin{tabular}{lc}
\hline \multicolumn{1}{c}{ Sub-bacia } & $\begin{array}{c}\text { Indicador } \\
\text { de Gestão }\end{array}$ \\
\hline Sub- bacia do Igarapé Apeú & 0,62 \\
Sub- bacia do Rio Bujarú & 0,56 \\
Sub-bacia do Médio Guamá Oeste & 0,53 \\
Sub-bacia do Médio Guamá Leste & 0,42 \\
Sub- bacia do Baixo Guamá & 0,40 \\
Sub- bacia do Igarapé Mãe do Rio & 0,37 \\
Sub- bacia do Alto Guamá & 0,29 \\
Sub- bacia do Rio Sujo & 0,26 \\
\hline
\end{tabular}

Org.: do Autor, 2018.

A bacia do rio Guamá é composta de maneira heterogênea por 19 municípios que apresentam percentuais em área (contida na bacia) de representatividade diverenciados: Acará (0,80\%); Ananindeua (0,15\%); Belém
(0,96\%); Benevides (0,51\%); Bonito (0,65\%); Bujarú (7,96\%); Capitão Poço (21,59\%); Castanhal (3,82\%); Concórdia do Pará (5,40\%); Garrafão do Norte (13,92\%); Inhangapi (3,87\%); Irituia (11,15\%); Mãe do Rio (3,38\%); Marituba (0,28\%); Ourém (3,45\%); Santa Izabel do Pará (4,78\%); Santa Luzia do Pará (5,48\%) ;São Domingos do Capim (3,83\%); e São Miguel do Guamá (8\%). Destes 14 apresentam suas sedes municipais contidas na área da bacia. Como os indicadores de gestão são referentes a atuação política no município como um todo, optou-se ponderar de maneira igual, uma vez que entende-se que as ações de gestão uma vez consolidadas vão ter reflexos distribuídos em todo território.

$\mathrm{O}$ procedimento adotado segue a premissa discutida por Carvalho (2014) ao afirmar que, a integridade das bacias hidrográficas deve conter uma compreensão sistêmica de sustentabilidade, sendo a água entendida em no conjunto das relações espaciais, com o planejamento ambiental integrado de bacias hidrográficas associado ao planejamento territorial.

Nota-se que a sub-bacia que apresenta maior valor quanto a este indicador é a do igarapé Apeú, a qual compõe os municípios de Castanhal e Santa Izabel do Pará que obtiveram valores acima de 0,50 e Inhangapi que teve um baixo indicador de gestão. Já as sub-bacias do rio Sujo e do alto Guamá apresentaram os menores valores, isto se dá devido os municípios de ambas possuírem indicadores abaixo dos 0,40 , caracterizando os municípios que precisam de ações mais 
imediatas para o fortalecimento da gestão ambiental.

\section{Índice de sustentabilidade hídrica}

A sustentabilidade hídrica foi calculada inicialmente por sub-bacia, onde foram enquadrados os resultados de acordo com cada indicador: hidrológico, ambiental, social e de gestão (Figura 2). A Figura 3 representa o tratamento dado as categorias de indicadores adotando a metodologia de clustering. As relações observadas espacialmente ou por agrupamentos indicam as similaridades existentes e os fatores de maior intervenção. A bacia pode ser visualizada em 3 grandes segmentos: Alto Guamá - Rio Sujo - Ig. Mãe do Rio; Médio Guamá - Ig. Apeú; Rio Bujarú Baixo Guamá.

Figura 2 - Índice de sustentabilidade hídrica com base nos indicadores ambiental, hidrológico, social e de gestão para as sub-bacias: I- Baixo Guamá; II- Igarapé Apeú; III- Rio Bujarú; IV- Médio Guamá setor oeste; V- Médio Guamá setor leste; VI- Igarapé Mãe do Rio; VII- Rio Sujo; VIII- Alto Guamá.

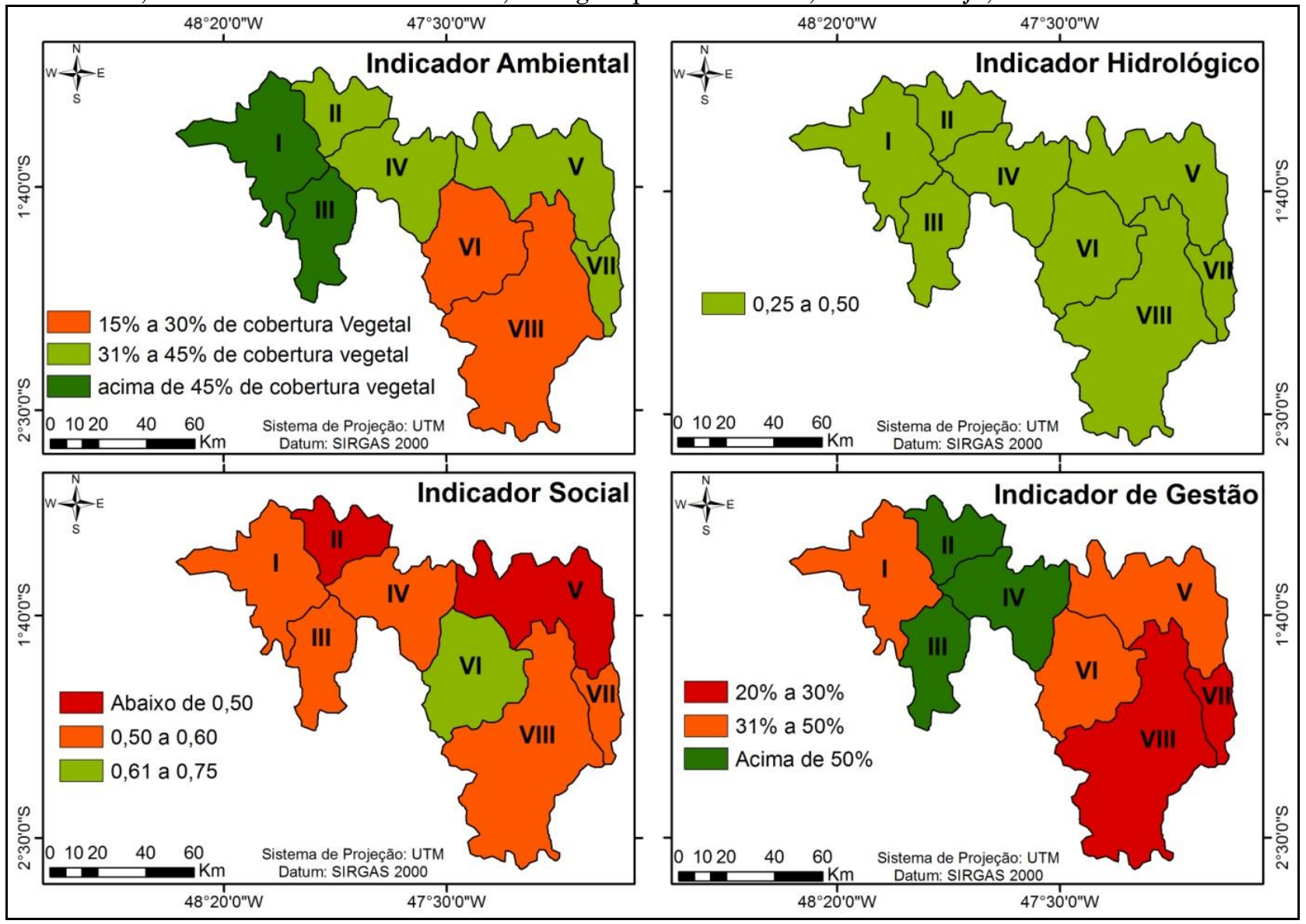

Org.: do Autor, 2018. 
Figura 3. (a) Resultado da análise hierárquica por agrupamento. (b) Representação da análise de agrupamento por matrizes de cores - Heatmaps.

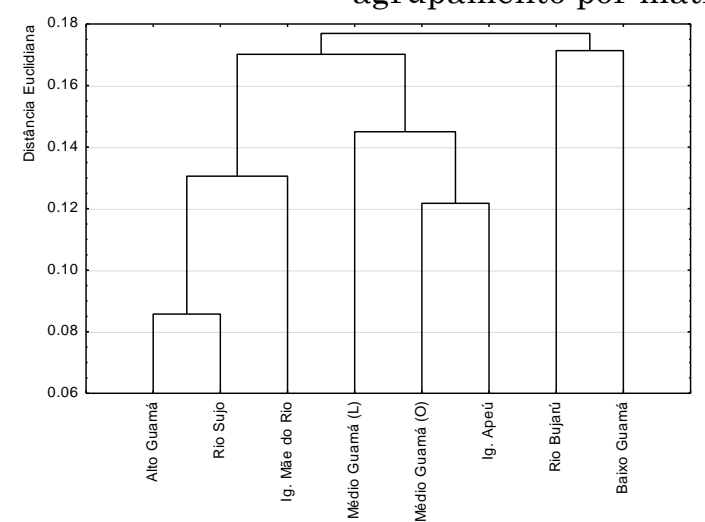

(a)

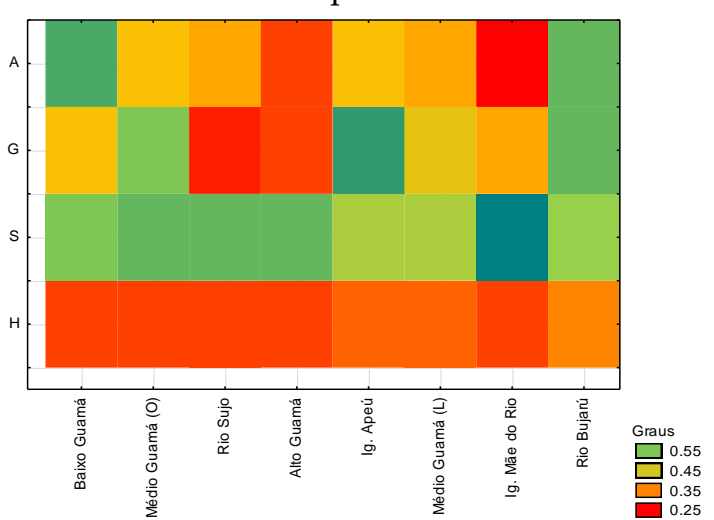

(b)

Org.: do Autor, 2018.

Os indicadores social (S) e de gestão (G) são os de maior responsabilidade pelas fragilidades observadas, pressionando principalmente as sub-bacias do Alto Guamá, Ig. Apeú e Ig. Mãe do Rio. O ISH é a média global dos quatro indicadores, e a partir desta análise de indicadores, foi possível obter os valores referentes ao índice de sustentabilidade hídrica para cada sub-bacia e seus respectivos desempenhos (Tabela 8). Com isso, a mesma obteve o valor de 0,54.

Tabela 8. Índice de sustentabilidade hídrica das sub-bacias.

\begin{tabular}{lcc}
\hline \multicolumn{1}{c}{ Sub-bacia } & ISH & Desempenho \\
\hline Sub-bacia do Baixo Guamá & 0,63 & Bom / potencialmente sustentável \\
Sub-bacia do Igarapé Apeú & 0,56 & Médio / intermediário \\
Sub-bacia do Rio Bujarú & 0,69 & Bom / potencialmente sustentável \\
Sub-bacia do Médio Guamá Oeste & 0,63 & Bom / potencialmente sustentável \\
Sub-bacia do Igarapé Mãe do Rio & 0,56 & Médio / intermediário \\
Sub-bacia do Médio Guamá Leste & 0,50 & Médio / intermediário \\
Sub-bacia do Rio Sujo & 0,50 & Médio / intermediário \\
Sub-bacia do Alto Guamá & 0,44 & Médio / intermediário \\
\hline
\end{tabular}

Org.: do Autor, 2018.

Maynard et al. (2017) obteve um valor de 0,66 para a bacia do rio Japaratuba (SE), com os indicadores mais fracos sendo o hidrológico e de gestão. Silva (2017) obteve o valor 0,53 para uma sub-bacia do rio PiranhasAçu $(\mathrm{RN})$, também identificando que de forma geral os indicadores vinculados aos aos aspectos socioeconômicos e de gestão apresentam uma considerável contribuição para a manutenção de média sustentabilidade.

Juwana et al. (2012), ao apresentar uma revisão de indicadores de sustentabilidade hídrica, destacam a influência que a componente "gestão" pode ter sobre as demais, onde condições desfavoráveis nos eixos "hidrológico" e "ambiental" podem ter seus 
impactos reduzidos com melhores condições de gerenciamento dos recursos hídricos.

Os valores do indicador social indicam a necessidade de buscar alternativas que contribuam para o melhoramento dos índices analisados, no intuito da obtenção de circunstâncias as quais apresentem uma situação ideal na qualidade de vida da população e garantia de qualidade nos serviços básicos. A gestão ambiental tem fundamental importância para a organização dos municípios e consequentemente, das subbacias. $\mathrm{O}$ investimento em ações voltadas a este critério representa uma estratégia benéfica para a manutenção das sub-bacias, além de fortalecer e conservar as relações de interdependência que ocorrem em seu território (MARTINS et al., 2010).

A média global obtida para a bacia evidencia a situação da bacia em relação à sustentabilidade hídrica, demonstrando a necessidade de definir programas de gestão hídrica capazes de potencializar o panorama apresentado, por meio de atuações mais responsáveis por parte das entidades reguladoras da gestão e demais setores da sociedade. A sustentabilidade hídrica sugere as condições necessárias para que as bacias tenham condições de recarga, compatível com a demanda existente, visando sempre um consumo igual ou inferior à sua capacidade de recuperação (TAMASAUSKAS et al., 2016).

Os indicadores analisados demonstram que o processo de uso e ocupação do território da bacia hidrográfica do Guamá tem alto potencial de interferir na sustentabilidade hídrica, já que estes exercem influência de caráter positivo ou negativo sobre a mesma. Onde a dinâmica evolutiva de uma paisagem passa a ser entendida por seus processos históricos nas diferentes escalas do tempo e pelas características ambientais predominantes, ambos os aspectos contribuem para o estabelecimento de um padrão de distribuição local (VIEIRA et al., 2007).

A análise evolutiva na perspectiva da sustentabilidade para a bacia do rio Guamá, pode ser avaliada por diversos marcos históricos, destacando-se a construção da Belém-Brasília (BR 010), responsável pelo surgimento de dezenas de vilas, povoados e cidades, destacando os da região NE do Estado (TAVARES, 2008). Dado este contexto, as formas de uso da terra tem marcado o nordeste paraense por um processo de descaracterização devido ao desmatamento; na região o percentual de alteração pelo antropismo já atinge quase $25 \%$, da mesma forma que cresceram os serviços de logística e de exploração dos recursos naturais, tais como: extração madeireira, agricultura de cortequeima e pecuária, sendo admitido um cenário formado por um mosaico diferentes graus de sucessão vegetal, culturas agrícolas e áreas de pastagem (CORDEIRO et al., 2017).

Em se tratando da bacia do rio Guamá, um fator que merece destaque pelo uso do seu território, é o fato desta abranger parte dos municípios da Região Metropolitana de Belém, com as cabeceiras das suas sub-bacias tendo como divisor de águas o eixo da BR-360, que interliga o Pará ao estado do Maranhão e a 
BR-010 que faz ligação com o sudeste do Estado. Desta forma, seus núcleos urbanos tem a capacidade de polarizar e influenciar um número significativo de cidades menores e articular relações de toda ordem, com a presença de centros urbanos de porte médio que integram a área metropolitana (TRINDADE Jr, 2011).

O uso da terra na bacia do Guamá confronta as perspectivas futuras de sustentabilidade hídrica para a região, quando observa-se o alto grau de urbanização, de concentração de atividades econômicas acompanhados pelo crescimento da pobreza e não superação dos problemas sociais (acesso à saúde, educação, habitação e saneamento básico) necessários para grande parcela da população (SILVA; SILVA, 2008).

\section{CONSIDERAÇÕES FINAIS}

O ISH foi mais representativo nas sub-bacias onde existe um maior escoamento superficial, maior concentração áreas vegetadas, a qualidade de vida foi significativa e a gestão mais fortalecida, sendo assim, os resultados obtidos indicaram que as sub-bacias do baixo Guamá $(0,63)$, do médio Guamá setor oeste $(0,63)$ e do rio Bujarú $(0,69)$ apresentam condições boas de sustentabilidade. Já as subbacias do alto Guamá $(0,44)$, igarapé Mãe do Rio $(0,56)$, rio Sujo $(0,50)$, médio Guamá setor leste $(0,50)$ e do igarapé Apeú $(0,56)$, encontram-se com desempenho médio. Com isso, a sustentabilidade hídrica da bacia do rio Guamá é intermediária com valor de 0,54.
Foi possível identificar que a pesquisa realizada em escala de bacia hidrográfica possibilitou a análise em conjunto dos aspectos hidrológicos, ambientais, sociais e de gestão. Considerando o contexto em que se encontra a bacia hidrográfica do rio Guamá, são necessárias medidas voltadas ao planejamento estratégico ligado à gestão e manejo da mesma, onde os gestores e demais setores da sociedade devem trabalhar de maneira mais eficiente para minimizar as pressões sobre a vegetação remanescente, para reforçar a capacidade institucional, e para melhorar a qualidade dos recursos e de vida da população, com o intuito de melhorar a sustentabilidade da bacia como um todo.

\section{AGRADECIMENTOS}

A Coordenação de Aperfeiçoamento de Pessoal de Nível Superior (CAPES) e ao Conselho Nacional de Desenvolvimento Científico e Tecnológico $(\mathrm{CNPq})$ pelo financiamento dado para o desenvolvimento da pesquisa.

\section{REFERÊNCIAS}

ARAGÓN, L. E. A dimensão internacional da Amazônia: um aporte para sua interpretação. R. NERA, n. 42, p. 14-33, 2018.

BARROSO, D. F. R.; FIGUEIREDO, R. O.; PIRES, C. S.; COSTA, F. F. Avaliação da sustentabilidade ambiental de sistemas agropecuários em microbacias do nordeste paraense a partir de parâmetros físicoquímicos. R. do Instituto Histórico e Geográfico do Pará, v. 2, n. 2, p. 56-68, 2015. https://doi.org/10.17553/23590831/ihgp.v2n2p56-68

BECKER, B. Amazônia: geopolítica na virada 
do III milênio. Rio de Janeiro: Garamond, 2009, 168 p.

BEZERRA, C. G.; SANTOS, A. R.; PIROVANI, D. B.; PIMENTEL, L. B.; EUGENIO, F. C. Estudo da fragmentação florestal e ecologia da paisagem na sub-bacia hidrográfica do córrego horizonte, Alegre, ES. Espaço \& Geografia, v. 14, n. 2, p. 257-277, 2011.

BLAIN, G. C.; BRUNINI, O. Análise comparativa dos Índices de Seca de Palmer, Palmer Adaptado e Índice Padronizado de Precipitação no estado de São Paulo. R. B. de Meteorologia, v. 22, n. 1, p. 105-111, $2007 . \quad$ https://doi.org/10.1590/S010277862007000100011

BLANC, I.; FRIOT, D.; MARGNI, M.; JOLLIET, O. Towards a New Index for Environmental Sustainability Based on a DALY Weighting Approach. Sustainable Development, v. 16, p. 251-260, 2008. https://doi.org/10.1002/sd.376

BÖHRINGER, C.; JOCHEM, P. E. P. Measuring the immeasurable - A survey of sustainability indices. Ecological Economics, v. 63, p. 1-8, 2007. https://doi.org/10.1016/j.ecolecon.2007.03.00 8

BOYACIOGLU, H. Dvelopment of a Water Quality Index Based on a European Classification Scheme. Water, v. 33, p. 101106 , 2007. http://dx.doi.org/10.4314/wsa.v33i1.47882

BRASIL. Instituto Brasileiro de Geografia e Estatística (IBGE). Censo demográfico $2010 . \quad$ Disponível em: <http://www.ibge.gov.br/home/>. Acesso em: 09 de Setembro de 2015.

BRASIL. Instituto Brasileiro de Geografia e Estatística (IBGE). Manual técnico de uso da terra: manuais técnicos em geociências. Brasília, DF: Instituto Brasileiro de Geografia e Estatística IBGE, 2013. $171 \mathrm{p}$.

BRASIL. Sistema nacional de informações sobre o saneamento (SNIS). Séries históricas. 2016. Disponível em: <http://www.snis.gov.br/>. Acesso em: 28 de setembro de 2017.

CABRAL, S. L.; CAMPOS, J. N. B.; SILVEIRA, C. S.; PEREIRA, J. M. R. O Intervalo de tempo para uma máxima previsiblidade da precipitação sobre o semiárido brasileiro. R. B. de
Meteorologia, v. 31, n. 2, p. 1-9, 2016. https://doi.org/10.1590/0102-

778631220130034

CALZAVARA, S. F.; FERNANDEZ, O. V. Q. Uso e ocupação do solo e número de curva (CN) na bacia hidrográfica do córrego Matilde Cuê, Marechal Cândido Rondon (PR). Geoingá, v. 7, n. 1, p. 185-209, 2015.

CARVALHO, J. R. M.; CURI, W. F.; CURI, R. C. Uso da análise multicritério na construção de um índice de sustentabilidade hidroambiental: estudo em municípios paraibanos. R. B. de Gestão e Desenvolvimento Regional, v. 9, n. 2, p. 3-26, 2013.

CARVALHO, J. R. M.; CURI, W. F.; CARVALHO, E. K. M. A.; CURI, R. C. Proposta e validação de indicadores hidroambientais para bacias hidrográficas: estudo de caso na sub-bacia do alto curso do Rio Paraíba, PB. Sociedade \& Natureza, v. 23, n. 2, p. 295-310, 2011.

CARVALHO, R. G. As bacias hidrográficas enquanto unidades de planejamento e zoneamento ambiental no Brasil. Caderno Prudentino de Geografia, n. 36, p. 26-43, 2014.

CHAVES, H. M. L.; ALIPAZ, S. M. F. An integrated indicator based on basin hydrology, environment, life, and policy: the watershed sustainability index. Water Resources Management, v. 21, n. 5, p. 883-895, 2007. http://dx.doi.org/10.1007/s11269-006-9107-2 CHUANG, Y. H.; YU, R. F.; CHEN, W. Y.; CHEN, H. W.; SU, Y. T. Sustainable planning for a coastal wetland system with an integrated ANP and DPSIR model for conflict resolution. Wetlands, Ecology and Management, v. 26, n. 6, p. 10151036 ,

2018. http://dx.doi.org/10.1007/s11273-018-9627-6

CORDEIRO, I. M. C. C.; RANGELVASCONCELOS, L. G. T.; SCHWARTZ, G.; OLIVEIRA, F. A. Nordeste Paraense: panorama geral e uso sustentável das florestas secundárias. Belém: Embrapa Amazônia Oriental, 2017, p. 19 - 58.

CORREIA, E. F. G.; RIBEIRO, G. P.; BAPTISTA, A. C. Modelagem hidrológica da bacia hidrográfica do rio Bengalas, Nova Friburgo, RJ, utilizando o potencial de geotecnologias na definição de áreas de 
risco à inundação. R. B. de Cartografia, v. 67, n. 6, p. 1183-1202, 2015.

CORTÉS, A. E.; OYARZÚN, R.; KRETSCHMER, N.; CHAVES, H.; SOTO, G.; SOTO, M.; AMÉZAGA, J.; OYARZÚN, J.; RÖTTING, T.; SEÑORET, M.; MATURANA, H. Application of the Watershed Sustainability Index to the Elqui river basin, North-Central Chile. Obras y Proyectos, v. 12, p. 57-69, 2012. http://dx.doi.org/10.4067/S0718. 28132012000200005

EMERSON, J.; ESTY, D. C.; LEVY, M. A.; KIM, C. H.; MARA, V.; SHERBININ, A.; SREBOTNJAK T. 2010 Environmental Performance Index. New Haven: Yale Center for Environmental Law and Policy, 2010, $87 \mathrm{p}$.

FAO. Water for Sustainable Food and Agriculture: a report produced for the G20 Presidency of Germany Rome: Food and Agriculture Organization of the United Nations, 2017, 33 p.

FARO, G. T. C.; GARCIA, J. I. B.; OLIVEIRA, C. P. M.; RAMOS, M. R. S. Application of indices for water resource systems stress assessment. B. J. of Water Resources, v. 24, e7, 2019. https://doi.org/10.1590/23180331.241920180106

FEITOSA, A.; FECHINE, J. A. L.; FERREIRA, C. W. S.; ARAÚJO, M. S. B. Modelagem dinâmica de escoamento superficial influenciando a susceptibilidade à erosão dos solos num município do semi-árido de Pernambuco. R. B. de Geomorfologia, v. $11, \quad$ n. $2, \quad$ p. $75-82,2010$. http://dx.doi.org/10.20502/rbg.v11i2.154

FERREIRA, A. V.; SÁNCHEZ-ROMÁN, R. M.; GONZÁLEZ, A. M. G. O. Temporal dynamic modeling for the assessment of water availability and its effects on sustainability of water resources at Boi Branco Sub-basin, SP, Brazil. Athens J. of Sciences, v. 3, n. 2 p. $137-154, \quad 2016$. https://doi.org/10.30958/ajs.3-2-4

FLORES-ALSINA, X.; GALLEGO, A.; FEIJOO, G.; RODRIGUEZ-RODA, I. Multiple-objective evaluation of wastewater treatment plant control alternatives. J. of Environmental Management, v. 91, p. 1193-1201, 2010. https://doi.org/10.1016/j.jenvman.2010.01.0 09
HTCF. Mission creek sustainable watershed indicators workbook. British Columbia: Habitat Conservation Trust Fund, 2003, 24 p.

JUWANA, I.; MUTTIL, N.; PERERA, B. J. C. Indicator-based Water Sustainability Assessment - a review. Science of the Total Environment, v. 438, p. 357-371, 2012.

https://doi.org/10.1016/j.scitotenv.2012.08.0 93

KEYANTASH, J. A.; DRACUP, J. A. An aggregate drought index: assessing drought severity based on fluctuations in the hydrologic cycle and surface water storage. Water Resources Research, v. 40, W09304, 2004. https://doi.org/10.1029/2003WR002610

MARQUES, R. V. Recursos hídricos no Brasil: um panorama histórico e institucional. $\mathbf{R}$. Tecnologia \& Cultura, v. 19 , n. 29, p. 1523, 2017.

MARTINS, A. A.; SOUZA, C. S. M.; FERREIRA, R. S.; SICILIANO, A. A importância da gestão ambiental com foco na sustentabilidade ambiental. Avesso do Avesso, v. 8, n. 8, p. 1-16, 2010.

MAYNARD, I. F. N.; CRUZ, M. A. S.; GOMES, L. J. Applying a sustainability index to the Japaratuba river wathershed in Sergipe State. Ambiente \& Sociedade, v. 20, n. 2, p. 201-220, 2017. https://doi.org/10.1590/18094422asoc0057r1v2022017

MUÑOZ-ROBLES, C.; REID, N.; TIGHE, M.; BRIGGS, S. V.; WILSON, B. Soil hydrological and erosional responses in patches andinter-patches in vegetation states in semiarid Australia. Geoderma, v. 160, p. 524-534, 2011. https://doi.org/10.1016/j.geoderma.2010.10.0 24

NARDO, M.; SAISANA, M.; SALTELLI, A.; TARANTOLA, T.; HOFFMAN, A.; GIOVANNINI, E. Handbook on Constructing Composite Indicators Methodology and User Guide. Paris, France: OECD Publications, 2008, 162 p.

NASCIMENTO, T. V.; FERNANDES, L. L. Mapeamento de uso e ocupação do solo em uma pequena bacia hidrográfica da Amazônia. Ciência e Natura, v. 39, n. 1, p. 170-178, 2017. 
PEREIRA, B. W. F.; MACIEL, M. N. M.; OLIVEIRA, F. A.; SILVA, H. A. S.; BRAGA, T. G. M.; FIGUEIREDO, D. B. Estrutura da paisagem da bacia hidrográfica do rio Peixe-Boi com base na fragmentação da vegetação. R. de Ciências Agrárias, v. 58, n. 2, p. 159-167, 2015.

PRATES, R. C.; BACHA, C. J. C. Os processos de desenvolvimento e desmatamento da Amazônia. Economia e Sociedade, v. 20, n. $3, \quad$ p. $601-636, \quad 2011$ https://doi.org/10.1590/S010406182011000300006

REBELLO, F. K.; SANTOS, M. A. S.; HOMMA, A. K. O. Modernização da agricultura nos municípios do nordeste paraense: determinantes e hierarquização no ano de 2006. R. de Economia e Agronegócio, v. 9, n. 2, p. 209-232, 2011.

RIBEIRO, C. R.; PIZZO, H. S. Avaliação da sustentabilidade hídrica de Juiz de Fora/MG. Mercator, v. 10, n. 21, p. 171188 , 2011 https://doi.org/10.4215/RM2011.1021.0012

RICKWOOD, C. J.; CARR, G. M. Development and Sensitivity Analysis of a Global Drinking Water Quality Index. Environmental Monitoring and Assessment, v. 156, n. 1-4, p. 73-90, 2009. https://doi.org/10.1007/s10661-008-0464-6

RIVERO, S.; ALMEIDA, O.; ÁVILA, S.; OLIVEIRA, W. Pecuária e desmatamento: uma análise das principais causas diretas do desmatamento na Amazônia. Nova Economia, v.19, n. 1, p. 41-66, 2009. https://doi.org/10.1590/S010363512009000100003

SANTOS, L. S.; MARTORANO, L. G.; BATALHA, S. S. A.; PONTES, A. N.; SILVA, O. M.; WATRIN, O. S.; GUTIERREZ, C. B. B. Imagens orbitais e termografia infravermelho na avaliação da temperatura de superfície em diferentes usos e cobertura do solo na floresta nacional do Tapajós e seu entorno-PA. R. B. de Geografia Física, v. 9, n. 4, p. 1234-1253, 2016.

SARTORI, A.; NETO, F.; GENOVEZ, A. Classificação hidrológica de solos brasileiros para estimativa da chuva excedente com o método do serviço de conservação do solo dos Estados Unidos Parte 1: Classificação. R. B. de Recursos
Hídricos, v. 10, n. 4, p.5-18, 2005. https://doi.org/10.21168/rbrh.v10n4.p5-18

SILVA, C. C. D.; ALBUQUERQUE FILHO, J. L.; OLIVEIRA, R. A.; LOURENÇO, R. W. Metodologia para análise do potencial de degradação dos recursos hídricos em bacias hidrográficas. Caderno de Geografia, v. 27, $\quad$ n. $\quad 50, \quad 2017$. https://doi.org/10.5752/p.23182962.2017v27n50p455

SILVA, D. D. C. Aplicação do índice de sustentabilidade de bacias hidrográficas no rio Piranhas-Açu a partir dos métodos multicritério e multidecisor. 2017. 312 f. Tese (Doutorado), Programa de Pós-Graduação em Recursos Naturais, Centro de Tecnologia e Recursos Naturais, Universidade Federal de Campina Grande, Paraíba, 2017.

SILVA, F. C.; SILVA, L. J. M. História regional e participação social nas Mesorregiões Paraenses. Paper do NAEA, n. 226 , p. 3 - 25, 2008.

SILVA, M.; NASCIMENTO, C. P.; COUTINHO, A. C.; ALMEIDA, C. A.; VENTURIERI, A.; ESQUERDO, J. C. D. M. A transformação do espaço amazônico e seus reflexos na condição atual da cobertura e uso da terra. Novos Cadernos NAEA, v. 16, n. 1, p. 229-248, 2013. http://dx.doi.org/10.5801/ncn.v16i1.608

SOOD, A.; RITTER, W. F. Developing a Framework to Measure Watershed Sustainability by Using Hydrological/Water Quality Model. J. of Water Resource and Protection, v. 3, p. 788-804, 2011. http://dx.doi.org/10.4236/jwarp.2011.311089

SOUSA, F. A. S.; DANTAS, F. R. C.; GUEDES, R. V. S.; MACEDO, M. J. H. Análise do Índice Padronizado de Precipitação para o estado da Paraíba, Brasil. Ambiente \& Água: An Interdisciplinary Journal of Applied Sciences, v. 5, n. 1, p. 204-214, 2010. http://dx.doi.org/10.4136/ambi.agua.130

SULLIVAN, C. A. Calculating a Water Poverty Index. World Development, v. 30, n. $7, \quad$ p. $1195-1210,2002$. https://doi.org/10.1016/S0305 750X(02)00035-9

SULLIVAN, C. A.; MEIGH, J. R.; GIACOMELLO, A. M. The Water Poverty 
Index: development and application at the community scale. United Nations Sustainable Development Journal, v. $27, \quad$ n. $3, \quad$ p. $189-199,2003$. https://doi.org/10.1111/1477-8947.00054

TAMASAUSKAS, P. F. L. F.; SOUZA, L. F. P.; LIMA, A. M. M.; PIMENTEL, M. A. S.; ROCHA, E. J. P. Métodos de avaliação da influência das áreas ripárias na sustentabilidade hidrológica em bacias hidrográficas no nordeste do estado do Pará. Caderno de Geografia, v. 26, n. 45, p. 172-186, 2016 . https://doi.org/10.5752/P.23182962.2016v26n45p172

TAVARES, M. G. C. A formação territorial do espaço Paraense: dos fortes à criação de municípios. R. Acta Geográfica, ano II, n. 3, $\quad 2008 . \quad$ p. $\quad 59 \quad-\quad 83$. https://doi.org/10.5654/actageo2008.0103.00 05

TEJADA-GUIBERT, J. A.; SETEGN, S. G.; STOA, R. B. Sustainable Development and Integrated Water Resources Management. In: SETEGN, S. G.; DONOSO, M. C. (Eds) Sustainability of Integrated Water Resources Management: Water Governance, Climate and Ecohydrology. Switzerland: Springer, p. 197-214, 2015.

TRINDADE, L. L.; SCHEIBE, L. F. Water management: constraints to and contributions of brazilian watershed Management Committees. Ambiente \& Sociedade. v. 22, e02672, 2019. https://doi.org/10.1590/18094422asoc20160267r2vu201912ao

TRINDADE Jr, S - C. C. Cidades médias na Amazônia Oriental das novas centralidades à fragmentação do território. R. B. Estudos Urbanos e Regionais, v. 13, n. $2, \quad 2011, \quad$ p. $\quad 135 \quad$ - 151. https://doi.org/10.22296/23171529.2011v13n2p135

VIEIRA, I. C. G.; TOLEDO, P. M. de; ALMEIDA, A. Análise das modificações da paisagem da Região Bragantina no Pará: integrando diferentes escalas de Tempo. Ciência e Cultura, v. 59, n. 3, p. 27-30, 2007.

VIEIRA, P. M. S.; STUDART, T. M. C. Proposta Metodológica para o Desenvolvimento de um Índice de Sustentabilidade Hidro-Ambiental de Áreas
Serranas no Semiárido Brasileiro - Estudo de Caso: Maciço de Baturité, Ceará. R. B. de Recursos Hídricos, v. 14, n. 4, p. 125136 , 2009. https://doi.org/10.21168/rbrh.v14n4.p125136

XU, Z. X.; JINNO, K., KAWAMURA, A.; TAKESAKI, S.; ITO, K. Sustainability analysis for yellow river water resources using the system dynamics approach. Water Resources Management, n. 16, p. 239-261, 2002. https://doi.org/10.1023/A:1020206826669

WATRIN, O. S.; CRUZ, C. B. M.; SHIMABUKURO, Y. E. Análise evolutiva da cobertura vegetal e do uso da terra em projetos de assentamentos na fronteira agrícola amazônica, utilizando geotecnologias. Geografia, v. 30, n. 1, p. 59-76. 2005.

WELSCH H. Constructing Meaningful Sustainability Indices. Applied Research in Environmental Economics, v. 31, p. 7-22, 2005. https://doi.org/10.1007/3-79081645-0_2

WILLET, J.; WETSER, K.; VREEBURG, J.; RIJNAARTS, H. H. M. Review of methods to assess sustainability of industrial water use. Water Resources and Industry, v. 21, 100110, 2019. https://doi.org/10.1016/j.wri.2019.100110

YOSHIMITANAKA, O.; DRUMOND JÚNIOR, M.; CRISTO, E. B.; SPEDO, S. M.; PINTO, N. R. S. Uso da análise de clusters como ferramenta de apoio à gestão no SUS. Saúde e Sociedade, v. 24, n. 1, p. 34-45, 2015. https://doi.org/10.1590/S010412902015000100003 